\title{
Publishing in Eye: The current status
}

Eye (2007) 21, 1-2. doi:10.1038/sj.eye.6702635; published online 27 October 2006

The primary aim of Eye, as the official journal of the Royal College of Ophthalmologists, is to provide the practicing ophthalmologist with information on the latest and most relevant clinical and laboratory-based research. Although the journal is primarily aimed at the practicing clinician, Eye contains material of interest to a wider readership, including optometrists, orthoptists, other health care professionals and research workers in all aspects of the field of visual science.

Eye has achieved a steadily rising impact factor in recent years, which has contributed to a tremendous increase in the number of manuscripts submitted to the journal submission rates have risen by almost $70 \%$ since 2003 when the journal first started accepting electronic submissions. Although we are flattered by the high demand, a high number of submissions pose several practical problems as we have limited capacity to publish the articles. The measurable effect of the high submission rate is that Eye's rejection rate has increased in recent years. In addition, the backlog of articles waiting to be published in print has increased, causing an increase in the mean time from acceptance to print publication.

In order to gradually eliminate the print backlog and to return to our stated aim of print publication within 3 months of acceptance, the Editors have felt the need to reiterate some of the following criteria for acceptance, which are currently being strongly enforced:

1. Eye encourages the submission of original clinical research articles or laboratory research articles (with clinical relevance) of interest to the journal's wide readership described above. This category includes prospective randomized or non-randomized trials, case control studies, cross sectional studies, and cohort studies. Case series (unless avoiding all the common pitfalls) and case reports are less encouraged.
I Rennie, AD Singh and AJ Lotery

2. We are continuing to solicit ideas for review articles from potential authors but will only consider them for publication if prior approval was obtained from the Editor. Once submitted, review articles will undergo the same rigorous peer review process as original research articles.

3. Authors who violate ethical issues such as duplications and plagiarism will continue to face disciplinary action. ${ }^{2}$

4. Effective immediately and until further notice, the appeal process has been suspended.

5. Articles submitted that do not conform to Eye's Instructions to Authors, or which have multiple grammatical and linguistic errors, will be rejected without further review.

Authors are strongly encouraged to adhere to the Instructions to Authors posted on the journal website (http://mts-eye.nature.com/) and to pay particular attention to the manuscript length, format, and style of writing. In brief: Abstracts should summarize the essential features of the paper in a logical and concise sequence (Aims/Purpose, Methods, Results, Discussion); Methods should provide enough detail for others to repeat the study; Results should be clear and precise; Discussions need not be a review of the literature but should focus on the interpretation and significance of the findings with objective comments that describe their relation to other work in the area.

Once these general guidelines are followed, referees can focus on evaluating the study design, analysis, validity, significance, and interpretation of the results. ${ }^{3}$ Ultimately, we serve you, our readers, who deserve and demand high-quality scientific articles so as to influence your decision-making and improve the care of our patients.

To ensure clarity for authors, the above guidelines have been incorporated into Eye's Instructions to Authors. Please visit http://mts-eye.nature.com for further information and to submit your article online.
Department of

Ophthalmology, Royal Hallamshire Hospital, Room K125, K Floor, Glossop Road, Sheffield S10 2JF, UK

Correspondence: IG Rennie, Tel: + 44114271 2902; Fax: +44114276 6381 . E-mail: i.g.rennie@ sheffield.ac.uk 


\section{References}

1 Jabs DA. Improving the reporting of clinical case series. Am J Ophthalmol 2005; 139: 900-905.

2 Albert DM, Liesegang TJ, Schachat AP. Meeting our ethical obligations in medical publishing: responsibilities of editors, authors, and readers of peer-reviewed journals. Arch Ophthalmol 2005; 123: 684-686.

3 Cummings P, Rivara FP. Reviewing manuscripts for Archives of Pediatrics \& Adolescent Medicine. Arch Pediatr Adolesc Med 2005; 156: 11-13. 